\title{
Electron-Positron Pair Production in Electro-Magnetic Field
}

\author{
Sadah A. Alkhateeb, Aisha Abdu Alshaery, Rawan Ali Aldosary \\ Mathematics Department, Faculty of Science, University of Jeddah, Jeddah, Saudi Arabia \\ Email: RALDOSARY0002.stu@uj.edu.sa
}

How to cite this paper: Alkhateeb, S.A., Alshaery, A.A. and Aldosary, R.A. (2022) Electron-Positron Pair Production in Electro-Magnetic Field. Journal of Applied Mathematics and Physics, 10, 237-244. https://doi.org/10.4236/jamp.2022.102017

Received: December 18, 2021

Accepted: January 26, 2022

Published: January 29, 2022

Copyright (C) 2022 by author(s) and Scientific Research Publishing Inc. This work is licensed under the Creative Commons Attribution International License (CC BY 4.0).

http://creativecommons.org/licenses/by/4.0/ (c) (i) Open Access

\begin{abstract}
At very high energies, pair production formation $\left(\gamma+\mathrm{N} \rightarrow e^{+} e^{-}\right)$exhibits a variety of intriguing properties. Analytically and quantitatively, the formation of Electron-Positron pairs in the Electro-Magnetic field of light nuclei has been calculated. In Ultra-Relativistic (UR) areas of incident photon energy, applying the resulting formulas to the energy distribution of the $\left(e^{-}, e^{+}\right)$operation. When we compare the results, we can observe that the Magnetic field of the target nucleus is more efficacious than the Electric field of the nucleus in the $\left(e^{-}, e^{+}\right)$operation. Furthermore, we can show that in Pair Production operation, the Differential Cross Section (DCS) owing to the target nucleus's Electric Quadrupole (EQ) and Magnetic Octupole (MO) are bigger than the Differential Cross Section (DCS) attributable to the target nucleus's Electric Charge (EC) distribution and Magnetic Dipole (MD).
\end{abstract}

\section{Keywords}

Pair Production, Positron, Differential Cross Section, The Bethe-Hitler Equation

\section{Introduction}

There are numerous particles in nature, each of which is accompanied by its field as it moves. They are split into Pheromones and Bosons. Pheromones are divided into Leptons and quarks. The Electron, Muon, and Tau are three charged Leptons, and neutrinos are three neutral Leptons [1]. Both the Electron and the Positron will be studied. The Electron was discovered in 1897 by J.J. Thomson, and it is still the prototypical elementary particle. Anderson saw Pair Production for the first time in 1932 when he exploited the operation to find the Positron [2]. Bethe and Heitler's work shaped our present theoretical understanding of Pair 
Production [3].

Nishina and others [4], Bethe, and Heitler [5] were the first to theorize the theoretical treatment of $\left(e^{-}, e^{+}\right)$Pair photon production in 1934. In 1936, Jaeger and Hulme [6] established that Pair Production Differential Cross Section (DCS) calculations produce better outcomes at high incident photon energy. Hubbell [7] provides a historical overview of the $\left(e^{-}, e^{+}\right)$by photons from $\mathrm{Di}$ rac's prediction of the position in 1928 until 2006. The (DCS) results for $\left(e^{-}, e^{+}\right)$ -Hubbell and Seltzer [8] revealed photon-based Pair Production.

Electron and Positron formation has been studied through high-energy collisions on the Nitrogen nucleus, with Atomic Number 7 and Mass Number 14, and is generated using Electric and Magnetic fields through high energies of incident photons (from 2 to $6 \mathrm{GeV}$ ). The results are given in tables and figures to show the difference in the energy distribution of $\left(e^{-}, e^{+}\right)$-Pairs. The obtained results are discussed in detail.

\section{Formulation of the Problem}

We are studying the effect of high energies on a light nucleus in the Pair Production operation.

\section{Research Objectives}

In this research, we present some new ideas for developing Electro-Magnetic operation and their various applications. This is done by studying the Electro-Magnetic (DCS) of nucleus Nitrogen and studying the extent of their impact on producing photons using high energies.

\section{Research Methodology}

The interaction of a photon with the nucleus of an atom produces Pairs of Electrons and Positrons. The $\left(e^{-}, e^{+}\right)$operation produced in the interaction of the $\gamma$-photon field with the field of nuclei (N), can be written: [9] [10] [11] (Figure $1)$.

$$
\gamma(k)+\mathrm{N}(Z e) \rightarrow e^{-}\left(p_{-}\right)+e^{+}\left(-p_{+}\right)
$$

Figure 2 depicts the Feynman Diagrams for the issue of Leptonic Pair Production in the Electro-Magnetic field of nuclei [12].

Where $q=p_{-}-k+p_{+}$indicates the momentum transmitted to the nucleus.

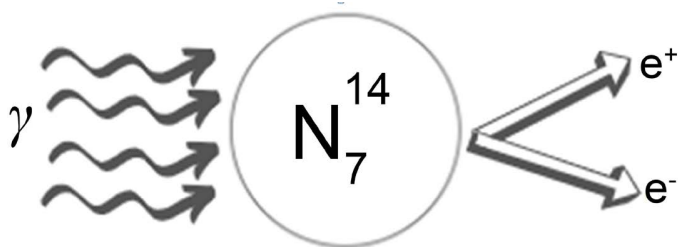

Figure 1. Simplified representation of the collision of a photon with a Nitrogen nucleus to the $\left(e^{-}, e^{+}\right)$. 

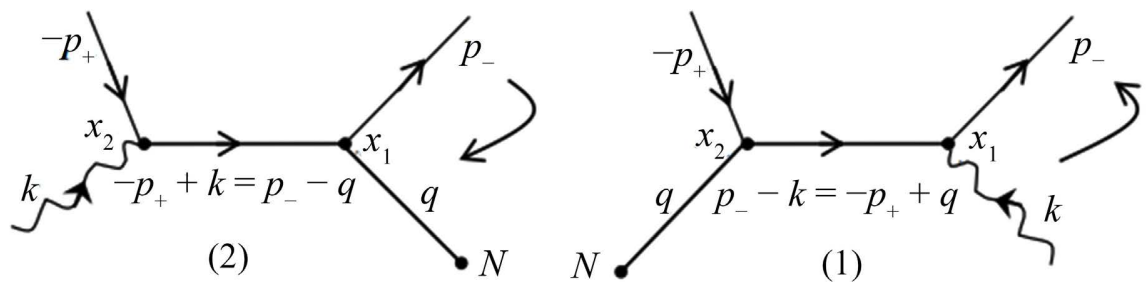

Figure 2. Feynman diagrams for the $\left(e^{-}, e^{+}\right)$pair production process.

This operation was studied by the scientist Bethe and Hitler. Therefore, the final form of the (DCS) of the operation of producing the Leptonic Pair of the nucleus is as follows: [3] [13] [14]

$$
\begin{aligned}
d B H= & \frac{Z^{2} \alpha^{3}}{(2 \pi)^{2}} \frac{\left|p_{+}\right|\left|p_{-}\right| d E_{+}}{\omega^{3}} \frac{d \Omega_{-} d \Omega_{+}}{|q|^{4}}\left[\frac{p_{-}^{2} \sin ^{2} \theta}{\left(E_{-}-p_{-} \cos \theta_{-}\right)^{2}}\left(4 E_{+}^{2}-q^{2}\right)\right. \\
& -\frac{p_{+}^{2} \sin ^{2} \theta_{+}}{\left(E_{+}-p_{+} \cos \theta_{+}\right)^{2}}\left(4 E_{-}^{2}-q^{2}\right) \\
& +2 \omega^{2} \frac{p_{+}^{2} \sin ^{2} \theta_{+}+p_{-}^{2} \sin ^{2} \theta}{\left(E_{+}-p_{+} \cos \theta_{+}\right)\left(E_{-}-p_{-} \cos \theta_{-}\right)} \\
& \left.-\frac{2 p_{+} p_{-} \sin \theta_{+} \sin \theta_{-} \cos \phi}{\left(E_{+}-p_{+} \cos \theta_{+}\right)\left(E_{-}-p_{-} \cos \theta_{-}\right)}\left(2 E_{+}^{2}-2 E_{-}^{2}-q^{2}\right)\right]
\end{aligned}
$$

It is the Bethe-Hitler equation for the (Electron-Positron) Pair Production operation, and it can be written in an abbreviated form so that it is applicable, using the following symbols:

$$
\eta=\left(\frac{Z^{2} \alpha^{3}}{4 \pi^{2}}\right) \frac{p_{+} p_{-} d E_{+}}{\omega^{3}}, \Delta_{0}=(1-\cos \theta)
$$

where $p_{+}=\left|\overrightarrow{p_{+}}\right|, p_{-}=\left|\overrightarrow{p_{-}}\right|$.

$\omega$ is the energy of the colliding photon where $\omega=E_{+}+E_{-}$. We can write Equation (1) as:

$$
\begin{gathered}
d\left(E, Z e, \mu_{1}, Q, \Omega\right)=d 1(E)+d 2(E)+d 11(E)+d 22(E) . \\
d 1(E)=8 \pi \eta \phi_{1}(E) d \Omega \\
d 2(E)=8 \pi \eta\left(\frac{\mu_{1}}{Z e}\right)^{2} a_{\mu} \phi_{2}(E) d \Omega \\
d 11(E)=8 \pi \eta\left(\frac{Q}{Z e}\right)^{2} a_{q} \phi_{11}(E) d \Omega \\
d 22(E)=8 \pi \eta\left(\frac{\Omega}{Z e}\right)^{2} a_{\Omega} \phi_{22}(E) d \Omega
\end{gathered}
$$

$\mathrm{Ze}, \mu_{I}, Q, \Omega$ are the (EC), the (MD), the (EQ), and the (MO) moments of the target nucleus. $\phi_{1}(E), \phi_{2}(E), \phi_{11}(E), \phi_{22}(E)$ in the case of high energies $E, E^{\prime} \gg m_{0} c^{2}$ [3] [13] [14]. 


$$
\begin{aligned}
& \phi_{1}(E)=\frac{1-\gamma}{4 k^{2} \omega^{2} \Delta_{0}}-\frac{1-\gamma^{2}}{8 \beta^{2} \Delta_{0}}+\frac{2 \gamma-7}{8 k^{2} \omega^{2} \Delta_{0}}-\frac{\varepsilon_{0}}{4 k^{2} \omega^{2} \Delta_{0}(1-\gamma)} \\
& -\frac{\varepsilon_{T}}{8 k^{2} \omega^{2} \Delta_{0}(1-\gamma)}\left[3 \gamma+\frac{k^{2} \omega^{2}}{\beta^{2}} \gamma(1-\gamma)^{2}\right] \\
& +\frac{L}{8 k^{2} \omega^{2} \Delta_{0}^{2}(1-\gamma)}\left[2+2(1-\gamma)^{2}+\gamma(2-\gamma) \Delta_{0}\right] \\
& \phi_{2}(E)=\frac{1}{2}\left[-3+\frac{k^{2} \omega^{2}}{\beta^{2} \Delta_{0}} \gamma\left(1-\gamma-\Delta_{0}\right)+\frac{\varepsilon_{0}}{1-\gamma}\left\{1-\gamma+\frac{1+(1-\gamma)^{2}}{\Delta_{0}}\right\}\right. \\
& \left.+\frac{\varepsilon_{T}}{\beta^{3}} k^{2} \omega^{2} \frac{\gamma^{2}\left(1-\gamma+\gamma \Delta_{0}\right)}{1-\gamma}+\frac{L}{2(1-\gamma)}\right] \\
& \phi_{11}(E)=k^{2} \omega^{2}(4-\gamma) \Delta_{0}-2 k^{2} \omega^{2}\left[1+(1-\gamma)^{2}\right]+\frac{k^{2} \omega^{2}}{\Delta_{0}}(1-\gamma)\left[1+(1-\gamma)^{2}\right] \\
& +\frac{\varepsilon_{0}}{2(1-\gamma)} k^{2} \omega^{2}\left[1+(1-\gamma)^{2}\right]\left(2-\Delta_{0}\right) \\
& \phi_{22}(E)=k^{4} \omega^{4}\left[\frac{20}{3 \Delta_{0}}(1-\gamma)^{3}\left\{1-(1-\gamma)^{2}\right\}-12(1-\gamma)^{4}\right. \\
& +2(1-\gamma)\left\{5+2 \gamma+7(1-\gamma)^{2}\right\}-4 \Delta_{0}\left(2-\gamma^{2}+\gamma^{3}\right) \\
& \left.-\frac{4}{3}\left\{6+(1-\gamma)^{2}\right\} \Delta_{0}+\frac{\varepsilon_{0}}{1-\gamma}\left\{1+(1-\gamma)^{2}\right\} \Delta_{0}\left(2+\Delta_{0}\right)\right]
\end{aligned}
$$

where:

$$
\begin{gathered}
\beta_{0}=\sqrt{(1-\gamma)^{2}+2 \gamma \Delta_{0}}, \beta=k \omega \beta_{0}, L=2 \ln \left[\frac{2 \omega(1-\gamma)}{\gamma}\right], \gamma=\frac{\omega}{E}=\frac{\varepsilon_{T}}{E} \\
\varepsilon_{0}=2 \ln [2 \omega(1-\gamma)], \varepsilon_{t}=\ln \left[\frac{\beta-\gamma+1}{\beta+\gamma-1}\right] \\
a_{\mu}=\frac{s+1}{3 s} \\
a_{q}=\frac{1}{180} \frac{(s+1)(2 s+3)}{s(2 s-1)} \\
a_{\Omega}=\frac{2}{4725} \frac{(s+1)(s+2)(2 s+3)}{s(s-1)(2 s-1)}
\end{gathered}
$$

are the $(\mathrm{MD}),(\mathrm{EQ})$, and the (MO) coefficients of the nucleus with spin.

\section{Results and Discussion}

The (EC) $d 1,(\mathrm{MD}) d 2,(\mathrm{EQ}) d 11,(\mathrm{MO}) d 22$, total Electric $d E$, and total Magnetic $d M$ Differential Cross Section(DCS) for the $\left(e^{-}, e^{+}\right)$using formulas for the energy distribution are obtained for the nucleus $\mathrm{N}_{7}^{14}$ and for different values of incident photon energies $\varepsilon_{\gamma}=(2 \mathrm{GeV}, 4 \mathrm{GeV}, 6 \mathrm{GeV})$, where $m=9.109558 \times 10^{-28}$.

From Table 1, we can get the following results: 
- The (DCS) $d 1, d 2$ for $\left(\mathrm{N}_{7}^{14}\right)$ nucleus is decreased with increasing energies.

- The (DCS) d11,d22 for $\left(\mathrm{N}_{7}^{14}\right)$ nucleus is increasing with increasing energies.

From Figures 3-8, we conclude that

- The (DCS) $d 1$ and $d 2$ for $\left(\mathrm{N}_{7}^{14}\right)$ nucleus are decreased with increasing energies for the $\left(e^{-}, e^{+}\right)$, which the (DCS) $d 11$ and $d 22$ for $\left(\mathrm{N}_{7}^{14}\right)$ nucleus are increased with increasing energies for the $\left(e^{-}, e^{+}\right)$.

Table 1. Differential cross-section of the energy distribution of the $\mathrm{N}_{7}^{14}$-nucleus.

\begin{tabular}{ccccc}
\hline$\varepsilon$ & $d 1$ & $d 2$ & $d 11$ & $d 22$ \\
\hline 2000 & $1.19712 \times 10^{-37}$ & $1.73779 \times 10^{-36}$ & $4.51215 \times 10^{-38}$ & $3.28019 \times 10^{-19}$ \\
4000 & $1.4964 \times 10^{-38}$ & $9.41793 \times 10^{-37}$ & $9.02431 \times 10^{-38}$ & $2.84168 \times 10^{-18}$ \\
6000 & $4.43377 \times 10^{-39}$ & $6.56291 \times 10^{-37}$ & $1.35365 \times 10^{-37}$ & $1.00201 \times 10^{-17}$ \\
\hline
\end{tabular}

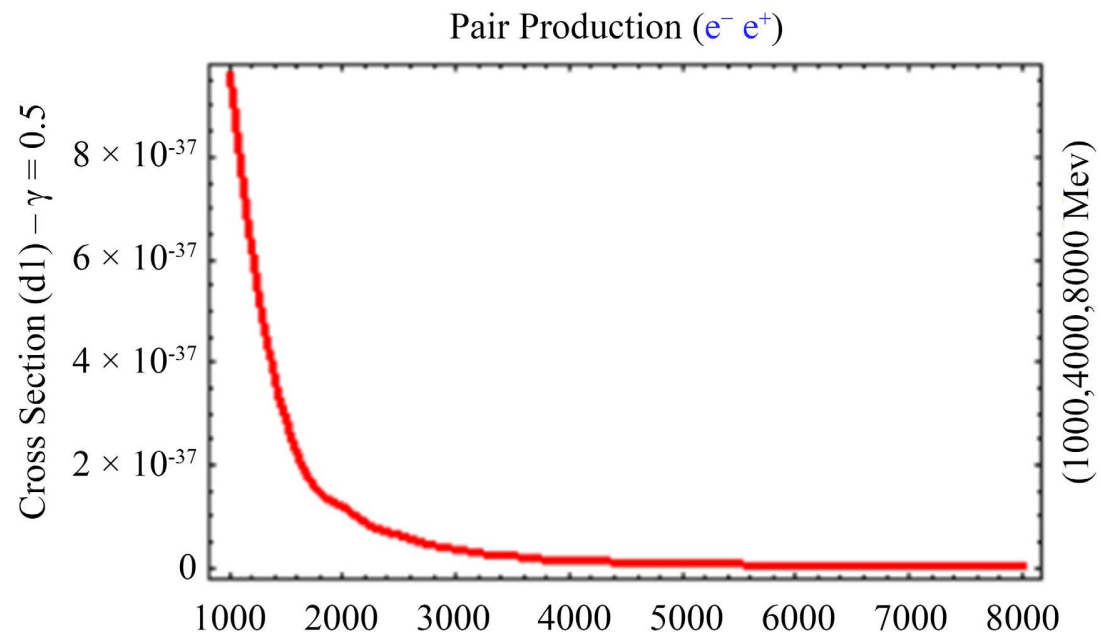

The Energy Degrees $\epsilon$

Figure 3. Electric charge $d 1$.

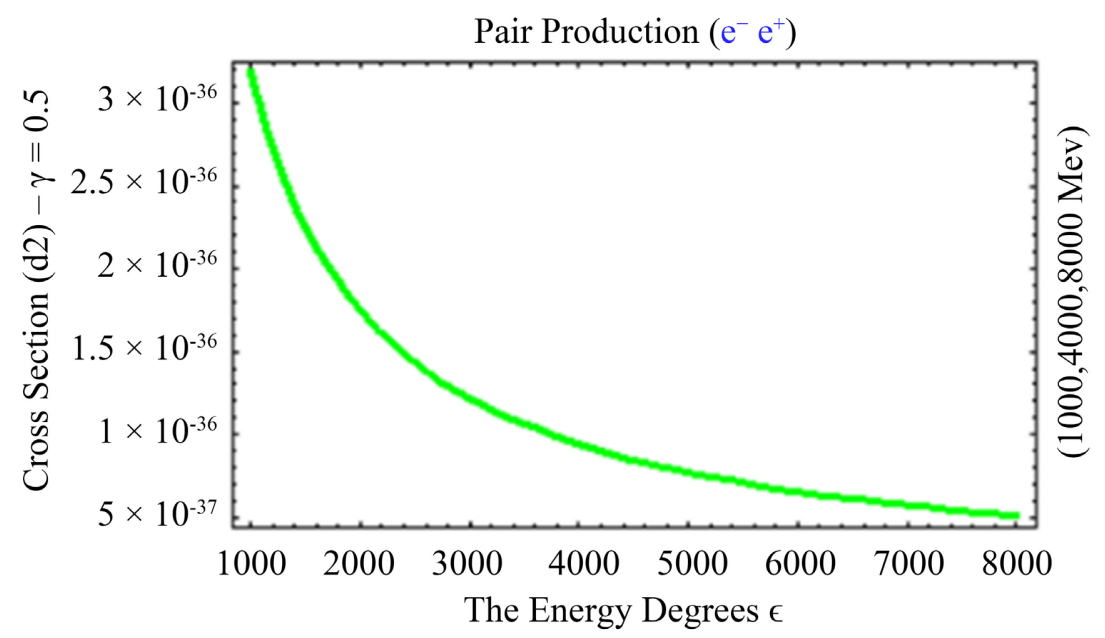

Figure 4. Magnetic dipole $d 2$. 
Pair Production $\left(\mathrm{e}^{-} \mathrm{e}^{+}\right)$

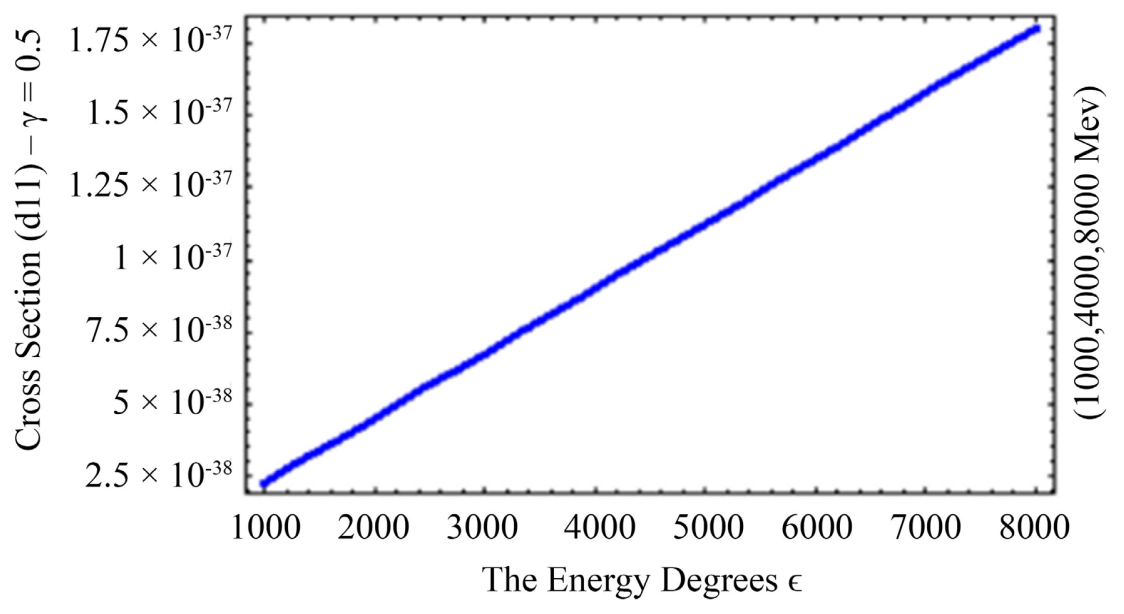

Figure 5. Electric quadrupole $d 11$.

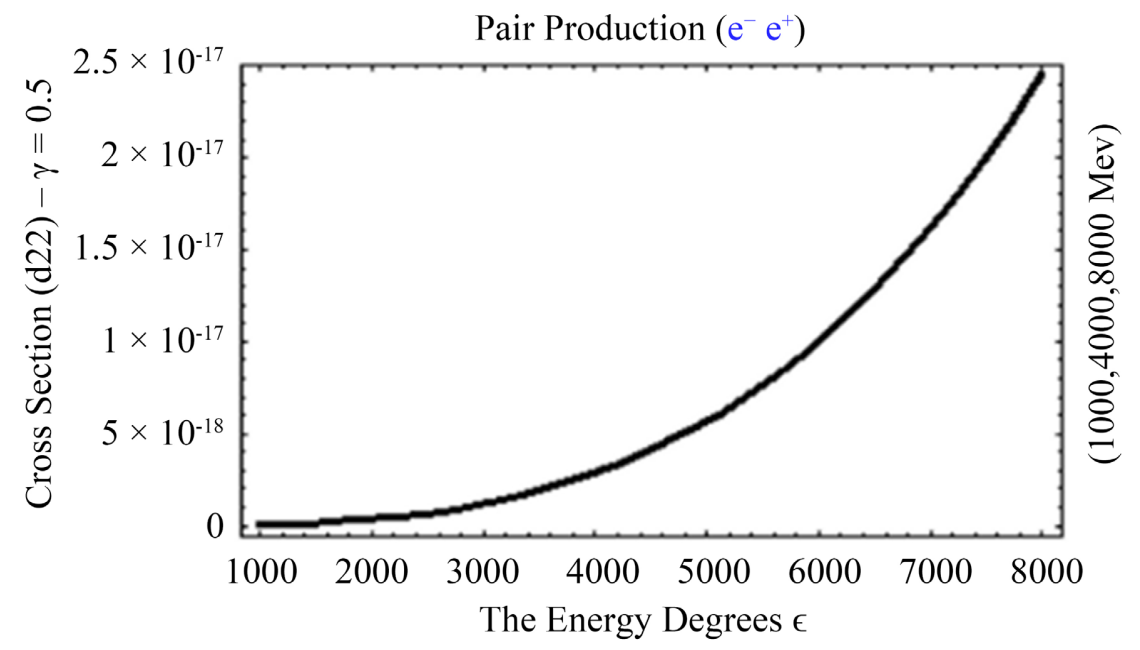

Figure 6. Magnetic octupole $d 22$.

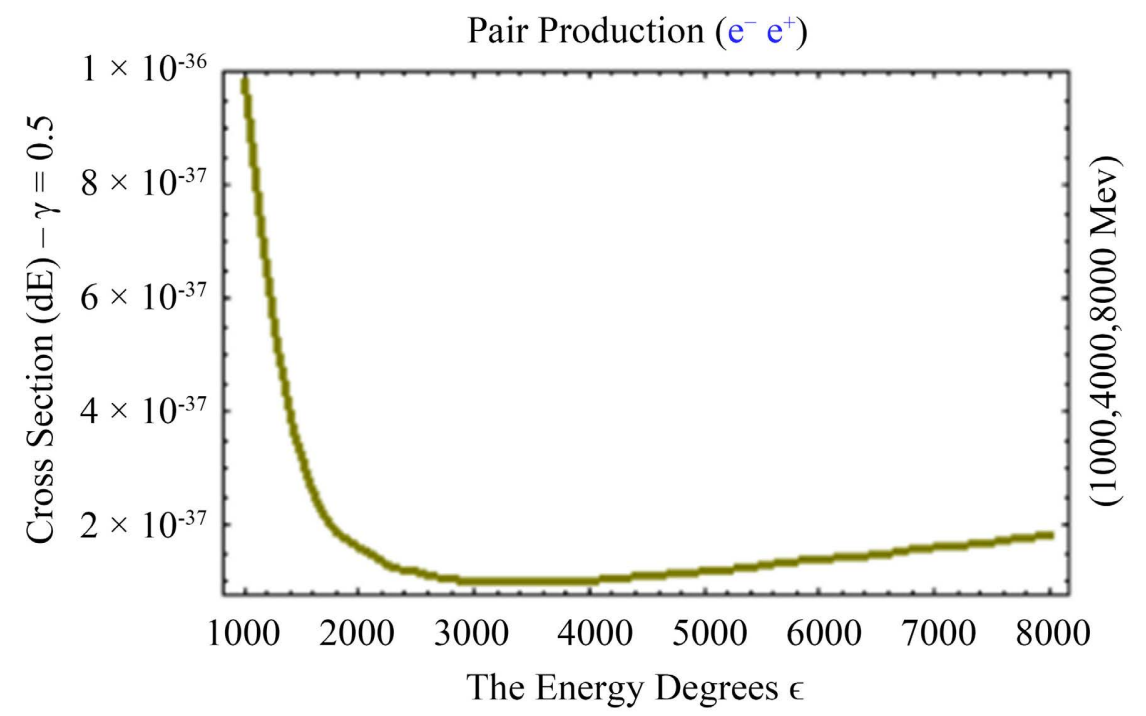

Figure 7. Total electric $d E$. 


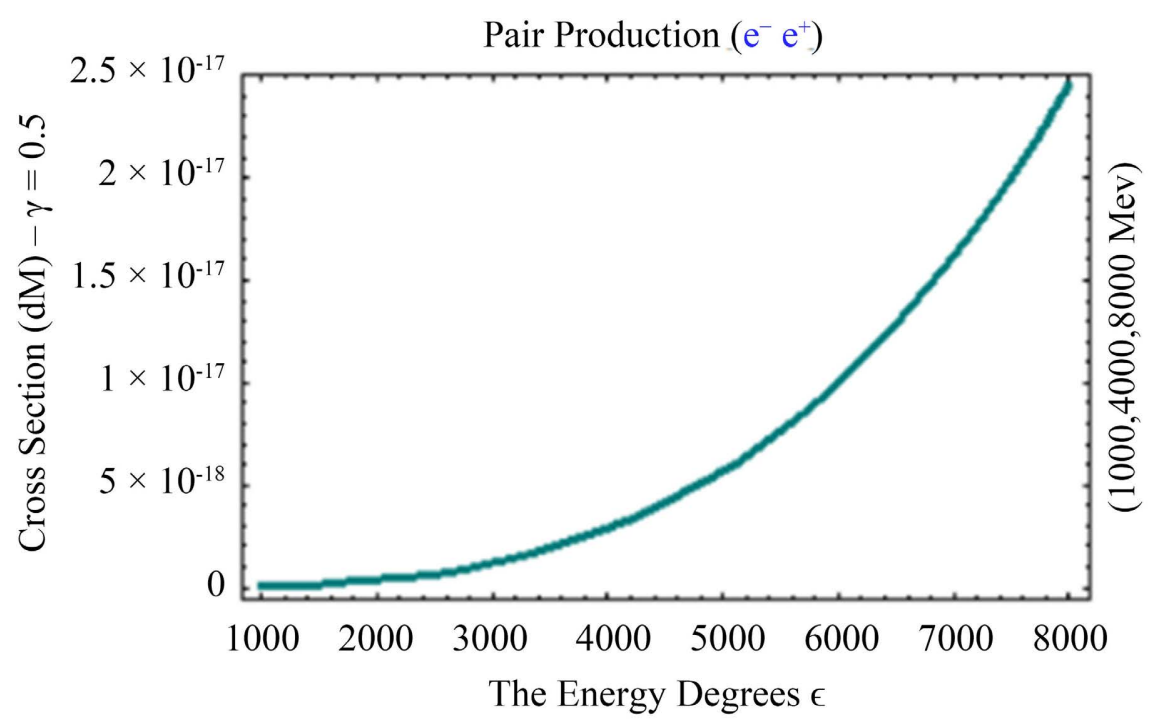

Figure 8. Total magnetic $d M$.

- The (DCS) of the $\left(e^{-}, e^{+}\right),(\mathrm{EQ}) d 11$, and (MO) $d 22$ is larger than the (DCS) of (EC) $d 1$ and (MD) $d 2$ of the target nucleus.

For the Total Electric $d E=d 1+d 11$ and the Total Magnetic $d M=d 2+d 22$ (DCS) we have also the following result:

- The (DCS) $d 11$ is more efficacious than the (DCS) $d 1$ for the (DCS) $d E$, i.e., $d E \approx d 1$.

- The (DCS) $d 22$ is more efficacious than the (DCS) $d 2$ for the (DCS) $d M$, i.e., $d M \approx d 22$.

- The Differential Total Magnetic Cross Section $d M$ for $\left(e^{-}, e^{+}\right)$-Pair Production is more efficacious than the Differential Total Electric Cross Section $d E$.

\section{Conclusion}

Compared to reference [13], we found that the operation of producing an $\left(e^{-}, e^{+}\right)$Pair in a Nitrogen nucleus of lighter mass is more effective than in a Sodium nucleus of higher mass. We conclude from this that the lower the mass number, the better the production of the pair. Moreover, we can see that in Pair Productions $\left(e^{-}, e^{+}\right)$, the Magnetic field of the target nucleus is more efficacious than the Electric field of the nucleus. The effect of the (QE) and (OM) (DCS) is more influential in the Lepton Pair's production than the (EC) and (MD).

\section{Perspectives}

We studied the creation of the $\left(e^{-}, e^{+}\right)$Pair for the Nitrogen nucleus through the relation of the energy of the photon falling on the nucleus and the Electro-Magnetic fields, and it can be studied in the future:

- The relation between the creation of the $\left(e^{-}, e^{+}\right)$Pair by the angle of the 
incident photon.

- The Production of the Lepton Pair by taking into account the polarization of the incident photons.

\section{Conflicts of Interest}

The authors declare no conflicts of interest regarding the publication of this paper.

\section{References}

[1] Greiner, W. and Reinhardt, J. (2008) Quantum Electrodynamics. Springer Science \& Business Media.

[2] Zettili, N. (2009) Quantum Mechanics Concepts and Applications. 2nd Edition, John Willy \& Sons Ltd., Chichester.

[3] Zhu, W. (2020) Improved Bethe-Heitler formula. Nuclear Physics B, 953, Article No. 114958. https://doi.org/10.1016/j.nuclphysb.2020.114958

[4] Nishina, Y., Tomonaga, S. and Sakata, S. (1934) On the Photoelectric Creation of Positive and Negative Electrons. Institute of Physical and Chemical Research.

[5] Bethe, H. and Heitler, W. (1934) On the Stopping of Fast Particles and on the Creation of Positive Electrons. Proceedings of the Royal Society A, 146, 83-112. https://doi.org/10.1098/rspa.1934.0140

[6] Jaeger, J. and Hulme, H. (1936) On the Production of Electron Pairs. Proceedings of the Royal Society A, 153, 443-447. https://doi.org/10.1098/rspa.1936.0013

[7] Hubbell. J. (2006) Electron-Positron Pair Production by Photons: A Historical Overview. Radiation Physics and Chemistry, 75, 614-623. https://doi.org/10.1016/j.radphyschem.2005.10.008

[8] Hubbell, J. and Seltzer, S. (2004) Cross Section Data for Electron-Positron Pair Production by Photons: A Status Report. Nuclear Instruments and Methods in Physics Research Section B: Beam Interactions with Materials and Atoms, 213, 1-9. https://doi.org/10.1016/S0168-583X(03)01524-6

[9] Klein, S.R. (2006) $e^{+} e^{-}$Pair Production from $10 \mathrm{GeV}$ to $10 \mathrm{ZeV}$. Radiation Physics and Chemistry, 75, 696-711. https://doi.org/10.1016/j.radphyschem.2005.09.005

[10] Chen, M.S., Muzinich, I.J., Terazawa, H. and Cheng, T.P. (1973) Lepton Pair Production from Two-Photon Processes. Physical Review D, 7, Article No. 3485. https://doi.org/10.1103/PhysRevD.7.3485

[11] Gong, C., Li, Z.L., Xie, B.S. and Li, Y.J. (2020) Electron-Positron Pair Production in Frequency Modulated Laser Fields. Physical Review D, 101, Article No. 016008. https://doi.org/10.1103/PhysRevD.101.016008

[12] Alkhateeb, S. (2012) The Leptonic Pair $e^{-} e^{+}, \mu^{-} \mu^{+}, \tau^{-} \tau^{+}$Production in the Photon Interaction with Electromagnetic Field of Light Nuclei. Journal of American Science, 8, No. 5 .

[13] Alkhateeb, S. (2020) Effect of Nuclear Magnetic Distribution on the Photon Production of Longitudinally Polarized Lepton-Pairs in the Field of $\mathrm{Na}_{11}^{23}$ and $\mathrm{Al}_{13}^{27}$ Nuclei. Thermal Science, 24, 139-147. https://doi.org/10.2298/TSCI20S1139A

[14] Obraztsov, I.V. and Milstein, A.I. (2021) Quadrupole Radiation and $e^{+} e^{-}$Pair Production in the Collision of Nonrelativistic Nuclei. Physics Letters B, 820, Article No. 136514. https://doi.org/10.1016/j.physletb.2021.136514 